\title{
Triple Triangulación Compleja: análisis de discursos de formación y empleo
}

\author{
Diana Amber' \\ Jesús Domingo" \\ 'Universidad de Jaén, Jaén - España \\ "Universidad de Granada, Granada - España
}

RESUMEN - Triple Triangulación Compleja: análisis de discursos de formación y empleo. Las dificultades económicas y la precariedad laboral sufrida por España en la crisis, generó un incremento del desempleo de los mayores de 45 años. En este contexto, este estudio pretende comprender el drama social presente en los diferentes discursos que giran en torno a ello mediante el uso de un nuevo diseño metodológico: la triple triangulación compleja. Con este diseño se contrasta el discurso de cuatro fuentes: mayores de 45 años, datos oficiales, medios de comunicación y expertos. Los principales hallazgos muestran discursos dispares con algunos puntos de encuentro entre las fuentes. Se concluye que muchos factores que intervienen en el desempleo de los mayores, rebasan el ámbito de la formación, siendo un problema multidisciplinar.

Palabras-clave: Formación. Empleo. Adulto. Discurso. Triangulación.

ABSTRACT - Complex Triple Triangulation: analysis of training and employment discourses. The economic difficulties and job insecurity suffered by Spain during the crisis produced an increase in unemployment among people older than 45 years. In this context, this study aims to understand the social drama that surrounds them - which is present in various discourses - by using a new methodological design known as complex triple triangulation. With this design the discourses of four sources are analysed: people older than 45 years, official datum, mass media, and experts. The main findings reveal certain differences and similarities between the discourses derived from these sources. It is concluded that a number of factors are involved in the unemployment rates of older people, with training overflow being a multidisciplinary problem.

Keywords: Training. Employment. Adults. Discourses. Triangulation.

Educação \& Realidade, Porto Alegre, v. 46, n. 1, e106754, 2021. 
Triple Triangulación Compleja

\section{Justificación ${ }^{1}$}

El contexto en el que se inicia y desarrolla este estudio se enmarca en la crisis económica que ha azotado a la sociedad y a su economía en los últimos años. Una crisis cuyas consecuencias no han afectado a todos con la misma intensidad, pues los colectivos más vulnerables han sufrido y siguen sufriendo sus efectos más directa y penetrantemente, incrementando las desigualdades entre la población de forma aberrante.

Entre las personas que han sido golpeadas con más dureza por la crisis económica, se encuentran los desempleados y desempleadas mayores de 45 años (Amber; Domingo, 2017a; Izquierdo; López-Martínez, 2013; Olesya, 2020), cuya tasa de desempleo se disparó durante los años en los que la crisis económica fue sentida con más fuerza en la sociedad y que aún no ha logrado recuperar los valores de antaño, sufriendo un nuevo azote a causa de la inestabilidad laboral acentuada por la pandemia (García-Calavia, 2020). Según la Encuesta de Población Activa del Instituto Nacional de Estadística (INE, 2020), en España, en el segundo trimestre de 2007, fecha previa a la crisis económica, 410.700 personas mayores de 45 años se encontraban en situación de desempleo, mientras que, para el mismo periodo de 2015 (año en el que la crisis económica estaba remitiendo), sumaban un total de 1.773 .300 personas sin empleo. Estos datos evidencian una cifra de desempleo que en pocos años fue cuadruplicada para estas personas y que, lamentablemente, en la actualidad apenas ha remitido, siendo de 1.184 .200 personas en 2020, en plena crisis sanitaria.

Muchas de estas personas en desempleo se formaron en sectores profesionales en declive que no pueden absorberlos en este momento, encontrándose a la deriva en un mercado de trabajo globalizado, digitalizado, altamente competitivo y caracterizado por la incertidumbre y la transitoriedad (Bauman, 2018; Beck, 2016). Con una identidad profesional plenamente formada, la caída emocional que supone el desempleo, afecta a todos los planos de la vida de las personas, suponiendo una brecha en su trayectoria profesional y vital que les obliga a replantearse todas sus experiencias, en definitiva, supone una crisis de identidad (Dubar, 2002).

En estas circunstancias sociales y personales, y con la edad como factor de exclusión del mercado laboral (Subirats, 2004), el acceso al empleo se dificulta enormemente. En respuesta a esta problemática, escasamente abordada por la literatura de investigación, con baja visibilidad social y mediática (Amber; Domingo, 2015), y para la que existen someras respuestas sociales o formativas, nace este trabajo de investigación, que busca analizar y comprender los discursos que se generan en torno a este proceso de exclusión socio-laboral. De este modo, y mediante el cruce de los discursos generados por las principales fuentes de información en torno a la cuestión, se propone desvelar los sentidos y significados atribuidos a la problemática, así como dar visibilidad a este conflicto social que afecta a las personas mayores de 45 años en 
desempleo. De forma paralela, este trabajo presenta un nuevo diseño metodológico, pertinente para el análisis de discursos complejos y multidimensionales: la triple triangulación compleja.

La presente investigación, por su alcance, amplitud y carácter multidisciplinar, es abordada desde el paradigma de la complejidad (Morin, 2008), mediante el uso de diferentes prismas y miradas que componen la realidad. Los entramados sociales que giran en torno a las circunstancias que envuelven a los desempleados mayores de 45 años, se expresan y manifiestan en discursos paralelos que abordan la realidad desde diferentes ópticas y cuyo compendio nos acerca a la comprensión de la problemática. En este sentido, no importa sólo adentrarse en las perspectivas sociales, que conjugan discursos diversos desde miradas ajenas, sino también, especialmente, en la mirada de la persona desempleada mayor, como profesional, miembro de una sociedad, dueña de su identidad y fruto de una trayectoria vital. Importa, además, averiguar cómo personas adultas y responsables de sus vidas sienten, perciben, valoran o dudan que necesitan formación para mejorar sus niveles de competencia, empleabilidad y permanencia en el controvertido momento actual por el que pasa el mercado de trabajo. Con ello aparecen claves comprensivas para enjuiciar las propuestas formativas recibidas, las que se les ofertan y las que se adecuarían significativamente a sus trayectorias personales y de vida laboral, con potencialidad real y viable de mejora.

Para atajar la problemática esbozada, con intención de dar respuesta a los objetivos planteados, se presenta a continuación la metodología que ha ayudado a abordar este trabajo, así como la estructura que organiza el laberíntico entramado de senderos que dan cuerpo a este estudio y los métodos utilizados para el análisis.

\section{Metodología}

Esta investigación enfrenta la realidad del desempleo de las personas mayores de 45 años entendiendo su complejidad, huyendo de la simplificación basada en el aislamiento y la abstracción de elementos, que denuncia Morin (2008).

La problemática del desempleo es sin duda una cuestión multidimensional, que puede ser abordada desde múltiples miradas, pues su complejidad es innegable. En respuesta a este entramado de interrelaciones que componen y enlazan la realidad que envuelve al sujeto en desempleo, este trabajo es planteado desde una mirada caleidoscópica. Desde este enfoque, se opta por una metodología en la que prima el enfoque cualitativo, pues es considerado pertinente para favorecer la comprensión y profundización en los fenómenos de estudio (Flick, 2018a). No obstante, conscientes de las singularidades de las metodologías cualitativa y cuantitativa y de su excelente complementariedad (Creswell, 2005), en los análisis previos que dan soporte a este estudio, ambas técnicas se combinan e integran en función de la naturaleza de la muestra. 
Triple Triangulación Compleja

Este complejo proceso de investigación implica el uso de una metodología cíclica (Flick, 2007), basada en un modelo de investigación circular, flexible y en continua adaptación, representado en la Figura 1. De forma que, los hallazgos de los primeros análisis van descubriendo nuevos intereses de estudio, que requieren de un nuevo muestreo, nuevas interpretaciones y estas a su vez crean nuevas inquietudes investigadoras en un proceso cíclico de continua revisión, perfeccionamiento y constante progreso. Recordemos el significativo verso de Antonio Machado que ilustra, aún sin pretenderlo, esta concepción de la metodología de investigación "Caminante, no hay camino, se hace camino al andar" (1965, p. 100).

\section{Figura 1 - Modelo Circular del Proceso de Investigación}

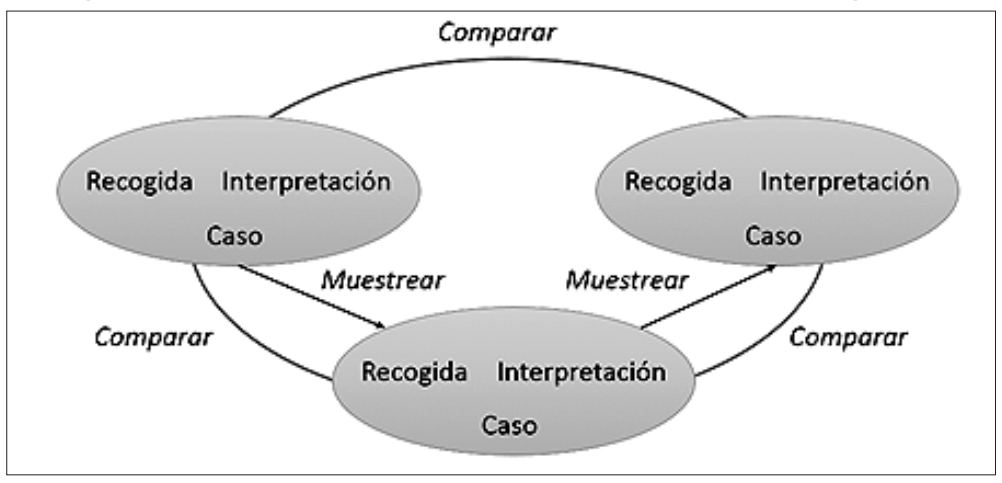

Fuente: Flick (2007)

\section{Estructura Metodológica}

Conscientes de que un solo método o fuente no puede captar todas las dimensiones de una realidad social, como garantía de validación se sigue un proceso de triangulación secuencial (Flick, 2018b), hasta alcanzar la saturación teórica (Denzin; Lincoln, 2011). El uso de diferentes fuentes y estrategias permite profundizar en el objeto de estudio y aportar fuerza al análisis (Heath, 2001; Tejada; Giménez, 2007). Este proceso es facilitado por las fluidas y variadas inquietudes emergentes de los estudios iniciales, que han ido encadenando los análisis de los que parte esta investigación y que se organizan en función de cuatro tipos de discurso, en una estructura metodológica generada a tal efecto que denominamos Triple Triangulación Compleja. Este nuevo diseño triangulado se caracteriza por recoger las miradas desde tres enfoques diferenciados, abordados a su vez desde una triple vertiente, en torno a un eje central. Los análisis individuales representados por cada uno de los vértices de los triángulos que componen la Figura 2, se relacionan entre sí y convergen en un punto central, pues todos ellos se posicionan sobre la misma problemática desde su propia idiosincrasia. Este punto central, esencia del estudio, supone el análisis principal, que a su vez 
es contrastado con todos los hallazgos obtenidos a partir de los demás estudios parciales, logrando la integración de los resultados que se presentan en este trabajo.

\section{Figura 2 - Esquema de Triple Triangulación Compleja}

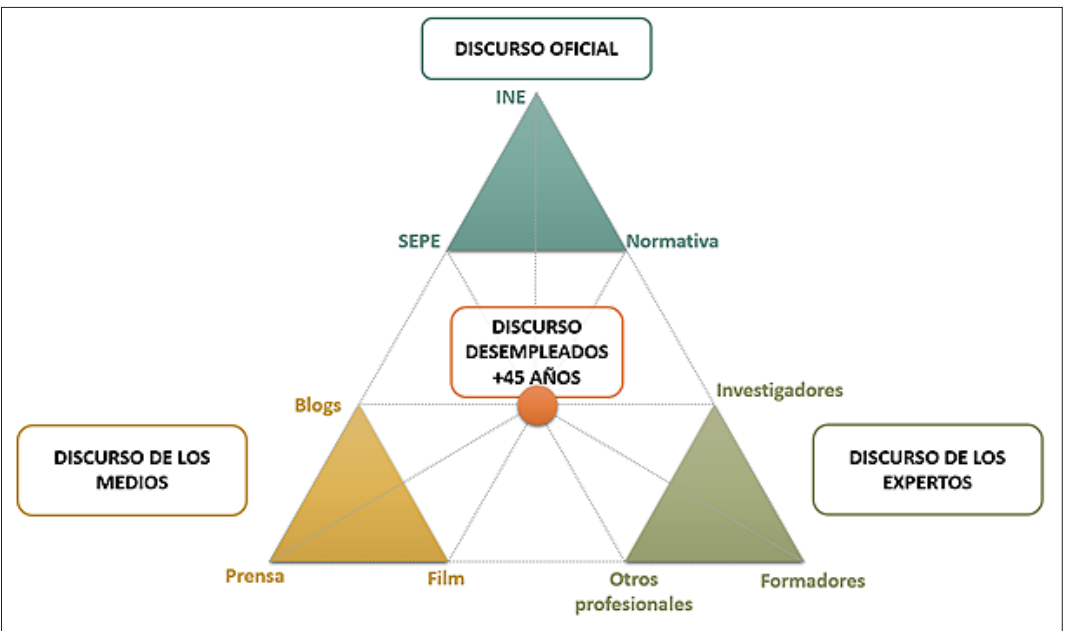

Fuente: Elaboración propia.

Esta estrategia de triangulación comparte con la triangulación de instrumentos y fuentes de información de Tejada y Giménez (2007) la multiplicidad de enfoques metodológicos y fuentes de información. La triangulación aportada por la diversidad de miradas que participan en este estudio y por la propia metodología cíclica, asegura el contraste de los datos y la consistencia de los análisis implementados. Además, el uso de diversas perspectivas permite analizar cómo es percibido el fenómeno de estudio desde diferentes enfoques (Stake, 2013).

Las diez fuentes utilizadas, recogidas en la Figura 2, se centran en cuatro tipos de discurso que se posicionan sobre la situación de desempleo de los mayores de 45 años:

- Discurso de las personas mayores de 45 años en desempleo.

- Discurso oficial: estadísticas oficiales del Instituto Nacional de Estadística (INE), estadísticas oficiales del Servicio Público de Empleo Estatal (SEPE) y normativa sobre formación y empleo.

- Discurso de los medios de comunicación: blogs, prensa y film.

- Discurso de los expertos: formadores, investigadores y otros profesionales.

Los cuatro tipos de discurso se abordan de forma conjunta en este trabajo en el apartado de integración de resultados, realizando un análisis triangular y contrastado entre ellos. Los datos fueron recabados y analizados de forma progresiva mediante un proceso cíclico en un periodo comprendido entre 2012 y 2016. 
Triple Triangulación Compleja

\section{Métodos de Análisis de la Información}

Este trabajo de triangulación se elabora a partir de los resultados hallados mediante diversos métodos de investigación y técnicas que se combinan y complementan en los diferentes análisis parciales de los discursos. Estos métodos se ajustan a la naturaleza de los datos y de la fuente. En la Tabla 1 se especifican los métodos, técnicas e instrumentos de recogida de datos, así como la muestra utilizada en cada uno de los análisis parciales.

Tabla 1 - Métodos, Técnicas e Instrumentos y Muestra de los Análisis Parciales ${ }^{2}$

\begin{tabular}{|c|c|c|}
\hline DISCURSO & MUESTRA & $\begin{array}{l}\text { MÉTODOS, TÉCNICAS E INS- } \\
\text { TRUMENTOS }\end{array}$ \\
\hline $\begin{array}{l}\text { Personas mayo- } \\
\text { res de } 45 \text { años } \\
\text { en desempleo }\end{array}$ & $\begin{array}{l}10 \text { personas desempleadas } \\
\text { mayores de } 45 \text { años }^{3} \text { (seleccio- } \\
\text { nadas mediante un proceso de } \\
\text { bola de nieve). }\end{array}$ & $\begin{array}{l}\text { Estudio de caso múltiple, análi- } \\
\text { sis biográfico-narrativo, análisis } \\
\text { de contenido (mediante entre- } \\
\text { vistas en profundidad). }\end{array}$ \\
\hline Oficial & $\begin{array}{l}\text { Estadísticas oficiales. } \\
\text { Normativa vigente sobre forma- } \\
\text { ción y empleo ( } 154 \text { documentos } \\
\text { de ámbito europeo, español y } \\
\text { andaluz). }\end{array}$ & $\begin{array}{l}\text { Análisis de contenido, análisis } \\
\text { documental y análisis secunda- } \\
\text { rio (meta-análisis). }\end{array}$ \\
\hline $\begin{array}{l}\text { Medios de co- } \\
\text { municación }\end{array}$ & $\begin{array}{l}12 \text { blogs seleccionados mediante } \\
\text { un grupo de expertos ( } 45 \text { post } \\
\text { específicos). } \\
7 \text { periódicos ( } 628 \text { noticias del } \\
\text { año 2014). } \\
\text { El film español "Los lunes al sol" } \\
\text { de Fernando León de Aranoa. } \\
\end{array}$ & $\begin{array}{l}\text { Análisis de contenido, análisis } \\
\text { lingüístico, análisis fílmico. }\end{array}$ \\
\hline Expertos & $\begin{array}{l}15 \text { expertos en formación y } \\
\text { empleo (seleccionados a partir } \\
\text { de un proceso de bola de nieve). }\end{array}$ & $\begin{array}{l}\text { Análisis de contenido (a partir } \\
\text { de entrevistas en profundidad). }\end{array}$ \\
\hline
\end{tabular}

Fuente: Elaboración propia.

Las estrategias y enfoques metodológicos empleados en los análisis parciales iniciales y los hallazgos obtenidos a partir de ellos, permiten un nuevo análisis contrastado de la información recabada, mediante el cruce de datos. Esta información inicial, es abordada en este estudio mediante el análisis del discurso desde su vertiente más crítica. La pertinencia de su análisis queda patente en el hecho de que los discursos muestran la realidad social, movilizan a las personas, las motivan, las conciencian, las dirigen, ejercen presión social, maquillan la realidad, son capaces de formar opinión pública. El discurso, tanto oral como escrito, goza de una importancia social tan acentuada que Van Dijk (2009, p. 158-159), en su conocido libro Discurso y poder asegura que “[...] aquellos que gozan de mayor control sobre más y más influyentes discursos (y sobre más propiedades discursivas), son también, según esta definición, más poderosos”. De manera que interesa conocer los entresijos que el discurso encierra a través de su análisis, siendo conscientes de la subjetividad del lenguaje y de su opacidad: 
Sabemos que el lenguaje no es transparente, los signos no son inocentes, que la connotación va con la denotación, que el lenguaje muestra, pero también distorsiona y oculta, que a veces lo expresado refleja directamente lo pensado y a veces sólo es un indicio ligero, sutil, cínico (Santander, 2011, p. 208).

El análisis del discurso realizado en este trabajo, se aborda esencialmente desde las trayectorias de vida de las personas desempleadas (analizadas desde el enfoque biográfico-narrativo) y su contextualización (Bolívar; Domingo, 2019), pues el discurso de los mayores de 45 años es el elemento central del estudio. Este planteamiento nos acerca a la comprensión de la causalidad de los acontecimientos sociales desde la perspectiva de los sujetos involucrados en ellos, a quienes se concede voz, pues siguiendo a Stake "[...] puede ser el mejor modo de adquirir una buena comprensión de los puntos débiles y fuertes y de avanzar hacia la mejora" (2006, p. 273).

A continuación, desde un abordaje multidisciplinar, interrelacionado y triangular de los discursos, mediado por su análisis crítico, se muestra la integración y discusión de los resultados de este trabajo, tras el procesamiento, relación e integración de los principales hallazgos obtenidos en los análisis parciales de cada uno de los cuatro discursos, estableciendo como eje central las historias de vida de las personas mayores de 45 años.

\section{Integración de los Resultados y Discusión}

En primer lugar, es necesario aclarar la significatividad del propio hecho de las escasas alusiones al colectivo en cuestión y a su problemática por parte de los medios de comunicación y el alto grado de generalización presente en los informes y estadísticas oficiales. Ambas cuestiones son indicadores de omisión y obviedad social del objeto de estudio, que es relegado a otros planos y eclipsado por temáticas de mayor impacto social.

En este apartado se realiza un contraste entre las fuentes de información analizadas, que muestra una visión conjunta, triangulada y comparada de los hallazgos parciales, mediada por el diseño de metodológico utilizado, y que a la vez permite una discusión con los principales planteamientos teóricos y resultados de otros estudios que trabajan en líneas paralelas. La complejidad de la realidad estudiada y las múltiples relaciones halladas entre sus elementos, hace pertinente la presentación de los resultados de forma integrada, pues todos los elementos se encadenan en un mar de complejas relaciones, cuyo desglose carece de sentido. Puesto que todos los discursos convergen en la situación de desempleo de los mayores de 45 años, se atiende a las tres grandes dimensiones emergentes de su discurso, como organizadores de los resultados: 1) Empleo; 2) Sentimientos y emociones derivados del desempleo y 3) Formación. Estas sirven como marco de referencia para la discusión y contraste con el resto de discursos que se pronuncian sobre ellos.

Educação \& Realidade, Porto Alegre, v. 46, n. 1, e106754, 2021. 
Triple Triangulación Compleja

\section{Empleo}

El empleo es percibido por los desempleados mayores como un símbolo de identidad. Pero tan solo en el discurso de estos destaca con fuerza el proceso de pérdida y ruptura de la identidad que el desempleo genera en las personas, especialmente en aquellas con una larga trayectoria profesional. Se evidencia en sus palabras una fuerte relación entre identidad y profesión, coincidente con la apreciada en el estudio de Lassus et al. (2015) sobre mayores en desempleo. De esta forma, en el discurso de los mayores, destaca el trabajo como símbolo de identidad, por encima de los demás sentidos del empleo. No obstante, como afirma Dubar (2002, p. 233) "[...] no todo el mundo prima la esfera profesional: la familia, la religión, la política o el ocio pueden representar ámbitos tan legítimos como ella de identificación “principal”. Este es el caso de Themis y Hestia, dos mujeres entrevistadas cuyo ámbito de identificación se asocia a la familia y no al empleo, persistiendo en ellas roles tradicionales de género.

Con gran influencia en la construcción de la identidad profesional, otra temática clave emergente de los discursos es la experiencia profesional. Esta, que se da por supuesta en los mayores de 45 años en el discurso de los medios de comunicación (especialmente en la prensa, que en ocasiones habla de ellos como profesionales maduros y con experiencia) y también en el discurso de los expertos, que la destaca como una de las potencialidades de este colectivo, no es siempre una baza para la persona desempleada. De los discursos se desprende la idea de que la experiencia puede ser entendida por los empleadores como un factor de resistencia, reivindicación y crítica o cuestionamiento de los procedimientos de la empresa. Algunos de los desempleados opinan que la experiencia no es valorada por el mercado actual, que las empresas prescinden de ella a fin de reducir costes, a pesar de que existe acuerdo en que esta es elemental en el desempeño profesional (Ruesga et al., 2014). Además, el contraste entre las percepciones sociales con los casos reales cuestiona la creencia social de que todos los desempleados mayores disponen de alta experiencia en un campo profesional, pues no todos ellos han disfrutado de contratos continuados y en el mismo sector a lo largo de su trayectoria profesional. En muchas ocasiones, estas trayectorias profesionales han sido discontinuas, especialmente en el caso de las mujeres entrevistadas, por motivos familiares. No obstante, es indudable que a lo largo de los años han acumulado aprendizajes y experiencias, tanto del ámbito profesional como de la vida. La madurez y experiencia del adulto, desde la visión de los expertos puede ser una fuente de aprendizaje para las nuevas generaciones, pues estos plantean como alternativa al empleo para las personas mayores la mentorización de los más jóvenes. En esta propuesta, los expertos coinciden con los desempleados en señalar la práctica de actividades generativas, como una opción de redefinición de la productividad de los mayores y de mejora de su bienestar, acorde con la tendencia a la generatividad propia del desarrollo adulto (Serrat et al., 2017; Zacarés; Serra, 2011). 
Las propuestas que se centran en la interacción entre las diferentes generaciones no se limitan a las iniciativas de mentorización y la promoción del aprendizaje intergeneracional, en el discurso crítico-reflexivo de los blogs analizados, se apuesta por la promoción de equipos intergeneracionales en las empresas, que aborden las problemáticas desde distintos enfoques generacionales, idea que podría generar una vía de acceso al mercado laboral de los mayores.

Entre las posibles estrategias de acceso al mercado laboral y de mantenimiento del puesto de empleo, los mayores de 45 años valoran especialmente la competencia profesional y las cualidades que le asocian (responsabilidad, compromiso, fidelidad hacia la empresa...), pues es lo que les permitió acceder y mantener su puesto de trabajo durante años. Curiosamente, en el resto de los discursos, este aspecto se obvia o se trata de soslayo. Esta llamativa discrepancia apoya los planteamientos teóricos que afirman que el mercado laboral actual se rige por nuevas reglas, que ignoran la lealtad y el vínculo entre empresa y empleado, pues sus notas fundamentales son el dinamismo, la movilidad y la transitoriedad de las relaciones laborales (Añez, 2016; Sennett, 2006). Por tanto, los nuevos discursos generados por los medios y por los expertos, se ajustan al discurso hegemónico actual y hacen hincapié en otros aspectos tales como la orientación, la cualificación y la actitud proactiva. Tan solo la normativa oficial parece gratificar la lealtad del trabajador hacia la empresa, otorgando prioridad de permanencia frente a los despidos. Iniciativa legal cuya efectividad se pone en duda, a juzgar por la cifra de desempleo en mayores ofrecida por las estadísticas oficiales.

La orientación profesional, esencial en el proceso de búsqueda de empleo para los expertos y muy presente en el discurso de los blogs, es planteada en el discurso de los mayores con falta de acuerdo entre ellos. Algunos denuncian la carencia de estos servicios y otros resaltan la percibida inutilidad de los mismos. No obstante, casos como Atenea y Apolo, reconocen su pertinencia especialmente como fuente de información sobre recusos y oportunidades, pues sienten que, como afirman Climent-Rodríguez y Navarro-Abal (2016), la orientación puede ayudar a las personas a redefinir su identidad profesional.

Las mayores coincidencias entre los discursos respecto a estrategias y recomendaciones que promueven el acceso al empleo coinciden en señalar la formación, la cualificación, la actitud proactiva del sujeto desempleado y el conocimiento de sí mismo como técnicas que lo favorecen (Alcoforado, 2013; Schömann, 2011; Thieme et al., 2015). Así, los mayores con baja cualificación son percibidos por los expertos como especialmente vulnerables y con baja esperanza de retorno al mercado laboral.

El emprendimiento, valorado por la literatura como posibilidad de reorientación profesional (Arráiz-Pérez et al., 2020), es la alternativa al trabajo por cuenta ajena para las personas desempleadas mayores de 45 años más recomendada por los expertos. Estos expertos, proponen además la necesidad de crear una cultura emprendedora e impartir formación que ayude a las personas a gestionar su propia empresa.

Educação \& Realidade, Porto Alegre, v. 46, n. 1, e106754, 2021. 
Triple Triangulación Compleja

Esto incentiva el emprendimiento por necesidad promovido en España (Martínez; Bogino, 2015). Por el contrario, los casos analizados (salvo Themis, que se lo plantea remotamente), rechazan el emprendimiento como opción laboral, especialmente a su edad, debido al riesgo que tendrían que asumir y al escaso tiempo laboralmente activo para recuperar su inversión inicial.

Otro de los aspectos que más lecturas suscita es la movilización social que, desde los desempleados, es propuesta únicamente por Zeus, como herramienta de defensa de los derechos laborales y mejora de las oportunidades de empleo. El discurso reivindicativo emerge también en el análisis de los blogs, generando una tipología de discurso contenido en ellos, que está conformado íntegramente por las palabras de los desempleados que se expresan por este medio. Medio que, para Zeus, ayuda a la reivindicación, pero no sustituye a la acción colectiva. La prensa coincide en incluir un matiz reivindicativo en algunas de sus noticias, en las que los sentidos del lenguaje que utilizan para referirse a los mayores apuntan en esta línea. Al igual que ocurre en los blogs, en todas las noticias de prensa con este tipo de discurso, el desempleado mayor juega un rol participativo en la definición de las propuestas o argumentos que la noticia presenta. Los medios, por tanto, facilitan la presencia de este modelo de discurso, que es incluido también en el film Los Lunes al Sol, representado por el personaje Santa (Amber; Domingo, 2016). En contraposición a la movilización como herramienta de éxito laboral, uno de los expertos se pronuncia y asegura que la actitud reivindicativa es contraria a los requerimientos del mercado laboral, que demanda flexibilidad y adaptación (Añez, 2016), por lo que puede ser vista por el empleador como un obstáculo o motivo de exclusión laboral, en línea con los resultados del estudio de Valdez-Montesdeoca y TapiaEspinoza (2020), que señalan la edad como un factor limitante desde la perspectiva empresarial. El discurso oficial, por su parte, apunta de forma indirecta hacia el aplacamiento de las agitaciones sociales que puedan ser emprendidas por los sujetos afectados por el desempleo. A tal efecto, los esfuerzos parecen dirigirse a la incentivación del individualismo (Beck; Beck-Gernscheim, 2003; Sennett, 2006) y a la desarticulación de acciones colectivas de los trabajadores (Bernad et al., 2011). El fracaso de las iniciativas de unión es claramente representado en el film analizado, que muestra los infructuosos esfuerzos de defensa de los derechos de los trabajadores emprendidos por sus protagonistas. Los mensajes de resignación, ocultos de forma implícita en los discursos, parecen bombardear a la sociedad. Las altas tasas de desempleo, mostradas por las estadísticas oficiales, son percibidas por Zeus como un recurso de sometimiento al mercado de trabajo, que lleve a aceptar ahora condiciones laborales en otros momentos impensables. Algunos mensajes contenidos en los blogs, aportan nuevos indicios de sometimiento, al señalar las ayudas y prestaciones sociales que promueven la dependencia y la precariedad, como un instrumento para aplacar y alimentar el silencio de los más vulnerables. 
Adentrándonos en los factores que acentúan el desempleo, la crisis económica se sitúa en las primeras posiciones de todos los discursos como principal justificación del desempleo que padecen los mayores de 45 años, junto a otros colectivos. En un mercado competitivo, con escasas oportunidades laborales, los mayores son percibidos como especialmente vulnerables, como víctimas de la situación económica, como queda patente en los principales sentidos del lenguaje de la prensa que a ellos se refiere. Los mayores son objeto de discriminación etaria como se reafirma en otros estudios (Arese, 2020; Gunderson, 2003; Weller, 2007; Olesya, 2020), siendo este un factor de vulnerabilidad que queda recogido por los medios de comunicación, destacado como obstáculo por los expertos y, coincidiendo con el estudio de Cheung et al. (2011), sentido especialmente por los propios desempleados. Esta discriminación, que supone una predisposición negativa del empresario, manifiesta en el discurso de expertos y desempleados, crea una barrera de exclusión difícilmente franqueable.

La exculpación atribuida a factores externos, tales como la crisis y la discriminación por edad, convive con otras percepciones que insisten en señalar al sujeto como responsable de su propia situación de desempleo. De este modo, del análisis lingüístico de los blogs se derivan una serie de aspectos tanto positivos (experiencia, talento, capacidad...) como negativos (carencias, errores, excusas...) que apuntan hacia el propio desempleado como merecedor de su propio destino profesional. Esta postura se ratifica en el discurso de la prensa, cuando uno de los sentidos del lenguaje empleados se refiere a ellos como profesionales estancados, con escasa motivación, baja cualificación o reacios al cambio. Las cifras estadísticas apoyan esta tendencia al mostrar bajos porcentajes de participación de los mayores de 45 años en actividades formativas. En esta línea de atribución, la mayoría de las recomendaciones indicadas por los expertos para el acceso al empleo de los mayores, se centran en acciones que debe emprender el sujeto, tales como mejora de su cualificación, generación de una red de contactos, estudio de idiomas, tecnología, etc. Las posiciones de los desempleados son dispersas en esta cuestión. Destaca la perspectiva individualista de Apolo, coincidente con el discurso de apoyo de los blogs, que señala al sujeto como principal motor del cambio y de la oportunidad laboral. Apolo, aún consciente de las problemáticas sociales, toma las riendas de su futuro profesional y marca una trayectoria de acción, en base a la creencia de que nadie lo hará por él. Otras posturas, como las de Atlas o Zeus, no acatan el individualismo que impera en el mercado y se oponen críticamente a él, considerando que el desempleo debería ser una cuestión social, que no debería enfrentar el individuo en solitario.

\section{Sentimientos y Emociones Derivados Del Desempleo}

Con todos los esfuerzos por aplacar la unión de los trabajadores y la movilización ciudadana comentados en el apartado anterior, no es de extrañar que el sentimiento más presente en el discurso de los 
Triple Triangulación Compleja

desempleados sea la resignación. Especialmente en tiempos de crisis, este sentimiento se acentúa, pues como aseguran Bernad, Martínez y Molpeceres (2011, p. 37) “[...] la situación de inestabilidad laboral acaba por asumirse como algo normal para una parte importante de la población”. No obstante, algunas voces entre los desempleados, ayudándose a veces de los medios de comunicación, como los blogs, se atreven a mostrar los sentimientos de reivindicación que les produce la abierta vulneración de sus derechos.

La tristeza y la desesperanza están también muy presentes en el discurso de los desempleados, pues la pérdida de empleo puede ser vivida como una experiencia especialmente dolorosa (Climent-Rodríguez et al., 2019), con consecuencias sobre el bienestar emocional y la salud (Álvarez-Gálvez; Suárez-Vergne, 2020). Los expertos que trabajan con ellos también son conscientes de su desolación y así lo expresan en su discurso. Este hundimiento emocional es recogido por los medios de comunicación, pues uno de los sentidos del lenguaje que usa la prensa al pronunciarse sobre los mayores en desempleo apunta a su desesperanza y desánimo. Más evidente aún es la desolación en los protagonistas de la película Los Lunes al Sol, que desde la visión del cine como espejo y modelo (Sell et al., 2014), muestra el desasosiego de los personajes en primera persona, con notas cargadas de dramatismo y a su vez de amarga realidad.

Muchos de los mayores de 45 años, admiten sentirse excluidos del mercado de trabajo, empujados hacia un lado y a la deriva. Sentimiento de exclusión que convive con la incertidumbre y con la impotencia ante una situación laboral que les deja al margen, con bajas probabilidades de lograr un empleo y, en muchos casos, sin opción a la jubilación. El sentimiento de impotencia se refuerza con los discursos de los medios que victimizan a las personas, que las dibujan como sujetos pasivos a merced de los cambios económicos y sociales, discursos que, al igual que las voces que los culpabilizan, anulan la autonomía e independencia de la persona (Caballol, 2002).

La culpa también está presente en el discurso de los desempleados, esta se deja ver en pocas ocasiones de forma explícita y con más frecuencia disfrazada de arrepentimiento, remordimiento o lamento por una mala decisión pasada. Los blogs, en sus discursos vivenciales también hacen eco de este sentimiento, que puede verse incentivado en los sujetos por los discursos sociales que los acusan.

Aunque en contadas ocasiones, la esperanza es el único sentimiento positivo que se desprende de los discursos. La esperanza aparece asociada habitualmente a individuos con nivel de formación medioalto en los sujetos entrevistados, al contrario que la desesperanza, más presente entre los menos formados. El discurso de los expertos apoya esta tendencia, pues entre las percepciones de los mayores desempleados que incluyen en su discurso, tan solo atribuyen posibilidades de reinserción a aquellos que gozan de alta especialización formativa y experiencial en su profesión. De forma paralela, perciben como vulnerables y sin esperanza a las personas con baja cualificación. 


\section{Formación}

La complejidad temática hace inabordable la problemática exclusivamente desde el marco de la formación, pues esta no puede atender a todos los aspectos que de ella se derivan. En línea con estos planteamientos, algunos de los desempleados, como Apolo (incluso tras su experiencia de éxito laboral con la realización de un curso), aseguran que la formación no es lo más importante, que muchas otras cuestiones intervienen en el proceso de logro o pérdida del empleo, aunque reconocen que una base formativa previa es imprescindible. La adquisición de esta base formativa, en la que los expertos insisten (siendo el desarrollo de competencias básicas la propuesta de formación de mayores más señalada por ellos), es poco valorada por los desempleados como necesidad de formación. Los desempleados entrevistados, en su mayoría, no consideran necesaria la adquisición de competencias básicas a su edad, pues ellos disponen de capacidades y habilidades que les hacen aptos para desempeñar la profesión a la que se han dedicado y muestran en su discurso su competencia para aprender a aprender, como lo han hecho diariamente en su puesto de empleo y en su vida cotidiana a partir de su experiencia o inquietudes personales. Tan solo dos mujeres, señalan la necesidad de desarrollar competencias básicas: Calíope refiriéndose concretamente a la competencia digital, en la que ha detectado una carencia personal; y Hestia, que defiende esta idea asociada a las enseñanzas regladas y obligatorias, sin extrapolarlo a la formación para el empleo.

La formación de los desempleados mayores está cargada de controversias. Algunos discursos coinciden en cuestionar la rentabilidad de la formación para el empleo de estas personas. Los propios expertos aseguran que, especialmente en tiempos de crisis económica, en los que los recursos están muy limitados, es posible que se esté reduciendo la inversión en formación para ese colectivo, pues les quedan pocos años en el mercado laboral para rentabilizar esa inversión, es decir, para lo que Zeus denomina sacarles el jugo. Otros informantes como Hiperión, también se cuestionan este asunto, coincidiendo con Calvo (2014, p. 355) en señalar que una de las barreras para la formación de este colectivo es "[...] la aparente escasa eficiencia económica del gasto en formación, tanto para el empresario como para el propio empleado”, debido a la cercanía del momento de jubilación.

Otra de las discusiones que la formación de mayores suscita, se esboza con las palabras de Orfeo, que afirma que a los mayores ya nos tienen por viejos. Estas palabras son clave para la comprensión de las dinámicas del mercado y para la orientación de la respuesta formativa para este colectivo. De este modo, las concepciones que señalan a la persona mayor como un sujeto con experiencia profesional y madurez, por tanto, con valor para el sistema productivo y susceptible de recibir formación para mejorar su empleabilidad, coexisten con aquellas que asocian al mayor con el adjetivo despectivo viejo (que tan solo Orfeo se atreve a pronunciar, pero cuyas connotaciones peyorativas aparecen en otros discursos). Este coloquial término los señala como prescindibles 
Triple Triangulación Compleja

para el mercado laboral y supone, por tanto, que la respuesta formativa pueda alejarse del marco laboral y acercarse al entretenimiento o al ocio. Algunos expertos proponen el ofrecimiento de actividades formativas orientadas al tránsito a la vida jubilar, mientras que los desempleados entrevistados cercanos a la edad de jubilación anhelan este retiro profesional y no parecen necesitar ayuda para dar este paso, aunque sí oportunidades y facilidades en su gestión.

Diferentes percepciones sobre la formación coexisten en los discursos analizados. Los medios de comunicación, los informes estadísticos, la normativa y los expertos coinciden en señalar a la formación cualificada como un valor para el mercado laboral, sin el cual, su acceso a este se complica. Sin embargo, no todas las opiniones de los informantes mayores apuntan en esta línea. Algunos sujetos, especialmente los más cercanos a la edad de jubilación muestran reticencias hacia ella, especialmente a procesos que impliquen una formación integral. Tan solo aceptarían iniciativas formativas que sirvan de perfeccionamiento profesional, pero que se apoyen en la base de su experiencia. Esta petición discrepa notablemente con las propuestas de los expertos, más centradas en procesos de formación integrales y en el desarrollo de competencias básicas en los sujetos, relegando a las últimas propuestas el desarrollo de competencias de perfeccionamiento profesional.

Las motivaciones que llevan a las personas a participar en actividades formativas son diversas. Para los desempleados entrevistados la formación es secundaria, priorizan el logro de un empleo, por lo que esta puede ser una forma de ocupar el tiempo o de divertimento. En esta línea uno de los expertos llega incluso a asegurar que la formación puede ser vista como un prozac para los desempleados, presunción que se confirma en el caso de Deméter, que asegura embarcarse en actividades formativas para huir de la depresión y el desánimo. Por el contrario, otros como Atlas, temen que, en su situación de desempleo, formarse pueda serle impuesto, interpretando la obligación de una formación permanente en la que el sujeto no puede decidir, como una condena (Hohmann, 2004).

Sin embargo, no todos los desempleados tienen la misma consideración sobre la formación. Coincidiendo con las cifras de los informes estadísticos oficiales, entre los entrevistados existe una mayor inclinación hacia la formación por parte de las mujeres y de las personas más jóvenes. Las estadísticas muestran también líneas de coincidencia con los casos en cuanto a la inclinación hacia la participación en actividades de formación entre los que ya tienen un mayor nivel formativo, consecuencia del llamado Efecto Mateo (Jiménez, 2009). Esta tendencia contrasta con el hecho de que la mayoría de las propuestas formativas que difunde la prensa se dirigen a profesionales sin cualificación, relacionando formación con carencia, en lugar de con progreso y perfeccionamiento. Esta percepción de los procesos formativos como compensatorios es compartida por los expertos. De ambos discursos (prensa y expertos) se desprende la conjetura de que muchas de las personas de esta edad tienen fuertes carencias formativas. Esta es contradicha por los infor- 
mes estadísticos, al mostrar que menos del 6\% de los desempleados de esta franja de edad no han completado sus estudios primarios y que el 16,01\% tienen estudios superiores (Amber; Domingo, 2017b). A su vez, también es refutada por los propios informantes y la diversidad formativa de los mismos, algunos de los cuales, como Themis, aseguran que los cursos de formación para el empleo en los que ha participado tienen un nivel demasiado bajo. Esta experiencia hace cuestionarse la necesidad de adaptar las iniciativas a los niveles de los participantes en ellas, propuesta muy destacada por los expertos.

En lo referente a los desafíos y retos que la formación para desempleados debe considerar, se detectan algunas analogías y discursos encontrados. Las mayores coincidencias entre expertos y desempleados, señalan la importancia del uso de una metodología práctica, el ajuste de la oferta formativa al mercado laboral y la enseñanza de contenidos útiles. Ambos apuntan también la importancia del diseño de una formación flexible que se ajuste a la disponibilidad del colectivo. En el caso de los desempleados, cabe destacar que los sujetos que lo proponen son mujeres, que alegan que deben conciliar la vida familiar con la formación.

El aumento de las oportunidades de formación es una propuesta altamente demandada por los desempleados y apoyada por los expertos. Dato curioso, al compararlo con el discurso de la normativa nacional, que hace gala de dar prioridad en la ejecución de políticas activas de empleo a los colectivos más vulnerables (RD 3/2011, de 18 de febrero). Medidas que parecen no tener gran calado en la población a juzgar por las experiencias de los informantes, que denuncian no encontrar posibilidades de formación, y por los expertos, que demandan mayores oportunidades formativas para los desempleados. Además, la narración de Deméter sobre las dos ocasiones en las que no es seleccionada para participar en un taller de formación que podría permitirle obtener la acreditación necesaria para trabajar fuera del ámbito de la economía sumergida, pone en cuestión la prioridad otorgada a los mayores de 45 años en la participación en actividades formativas, por la pertenencia a un colectivo con mayor dificultad de inserción laboral (RD 694/2017, de 3 de julio).

Entre las propuestas para la mejora de la formación dirigidas a los mayores, los desempleados también se posicionan sobre las características profesionales que esperan del profesorado que la imparta. Le exigen, por supuesto, competencia profesional y conocimiento, pero también demandan una relación que permita el intercambio de opiniones y la participación, que admita la discusión sobre las líneas temáticas y procedimientos, generando una relación entre iguales, como adultos y profesionales, en la que la frontera entre docente y discente se diluya, y el profesorado actúe como mediador del conocimiento, guía para el aprendizaje. Frente a esta demanda de los adultos, las percepciones de los formadores sobre sus alumnos maduros, suelen poner énfasis en la voluntad y el esfuerzo de estos para enfrentar la formación y en su disposición a mejorar, visiones que los catalogan como buenos alumnos, 
Triple Triangulación Compleja

pero que no dan a entender el trato entre profesionales que los mayores reclaman. Algunos de los expertos aseguran que es necesaria una mayor especialización del profesorado que forme a adultos, conscientes de la dificultad de abordar grupos tan diversos. No obstante, los formadores se enfrentan al reto cada vez más frecuente de impartir docencia a adultos en establecimientos, grupos y con planes formativos diseñados para otras generaciones.

Los expertos abogan por el fomento y la facilitación de la acreditación a partir de la experiencia profesional, acentuando que se trata de una cuestión de justicia social. Aunque el logro de acreditaciones a través de la experiencia no es referido por los desempleados entrevistados, los informantes más jóvenes sí hacen alusión a la acreditación de los procesos formativos como una de sus motivaciones para embarcarse en formación, pues consideran que las certificaciones pueden abrir puertas laborales.

Las implicaciones que se derivan de este trabajo rebasan el ámbito de la formación para el empleo, y se expanden hacia otros ámbitos y contextos educativos que trascienden al propio colectivo. En este sentido, los expertos señalan la importancia de la intervención educativa en etapas anteriores, que prevengan el edadismo. A su vez, destacan la necesidad del desarrollo de una economía solidaria desde el ámbito de la educación social y asocian la educación de los mayores con la de las nuevas generaciones, incidiendo en la influencia que estos ejercen sobre su descendencia. Estas implicaciones, con poca relevancia en los discursos mediáticos, tienen puntos de encuentro con el discurso de los adultos, especialmente en lo que atañe a la necesidad de la transformación de la cultura empresarial, a fin de invertir la predisposición negativa del empleador hacia la contratación de mayores.

\section{Conclusiones}

El uso de la triple triangulación compleja como diseño metodológico para el análisis de realidades complejas, ha facilitado el contraste de miradas y enfoques presentes en los discursos en torno a la problemática tratada, pudiendo extraer algunas conclusiones generales y cuestiones para su reflexión y debate.

En primer lugar, se destaca que la formación no es la solución única y absoluta al desempleo de los mayores de 45 años. En la exclusión laboral de este colectivo coexisten muchos más factores, mediados por la edad (Salvador; Sampietro, 2020), que se desbordan del alcance de la disciplina educativa y se vierten hacia otros ámbitos como la sociología, la psicología, la política o la economía. La formación es, sin duda, necesaria y es un empuje que ayuda a frenar el proceso de exclusión laboral y social; especialmente, si esta tiene continuidad a lo largo de la vida y permite la actualización profesional (Pochmann, 2012). Sin embargo, como reivindican muchos de los informantes de este estudio y afirma Sánchez (2009, p. 166) "La lucha contra la exclusión social se debe hacer a través de la educación y la formación, pero también a través del empleo remunerado". 
A su vez, la experiencia profesional también genera controversias, pues puede ser percibida como un obstáculo o como un valor para la inserción laboral. La tradicional valía de la experiencia profesional para el acceso al empleo se pone en cuestión para los desempleados mayores de 45 años. En el actual mercado de trabajo la alta experiencia laboral puede ser entendida como un indicativo de inflexibilidad, resistencia al cambio y como una fuente de conflictos y rebelión en la empresa. La experiencia tiene además un coste económico que muchas empresas no están dispuestas a asumir (Valdez-Montesdeoca; Tapia-Espinoza, 2020).

Por otra parte, el desempleo en esta franja de edad supone una ruptura con la vida profesional anterior, que repercute en la identidad de la persona y tiene fuertes connotaciones emocionales (ClimentRodríguez et al., 2019). El plano emocional emerge con fuerza ante la situación de desempleo, especialmente en aquellas personas con una larga y estable trayectoria profesional. La incertidumbre asociada al desempleo se contrapone con la estabilidad característica de esta etapa de la vida (Erikson, 2000), poniendo en jaque una identidad profesional ya creada (Dubar, 2002). El continuado esfuerzo personal que ha valido previamente para alcanzar progresivamente logros profesionales que se sienten propios y merecidos, es percibido como inútil, pues el desempleo les ha despojado de los frutos de su esfuerzo, creando sentimientos de defraudación.

Por todo ello, es necesario plantearse el desenfrenado sistema productivo actual, basado en la obtención del máximo beneficio al menor coste económico (Rodríguez-Rodríguez, 2012). Hemos de apostar por un desarrollo económico sostenible, que persiga el bien común (Felber, 2012), ayudándonos del fuerte poder transformador de la educación desde los primeros niveles formativos, con la convicción de que no es posible (ni recomendable) la producción y el consumo indefinido.

Recibido el 23 de agosto de 2020 Aprobado el 26 de diciembre de 2020

\section{Notas}

1 Este artículo está elaborado a partir de la tesis doctoral titulada "Mayores de 45 años en desempleo. Historias silenciadas y desafíos de la formación" y financiada mediante el programa de Formación del Profesorado Universitario (FPU) del Ministerio de Educación, Cultura y Deporte de España.

2 La selección de las muestras utilizadas, así como los procedimientos de recogida, codificación y análisis de la información, fueron concretados y definidos para cada discurso específico, en función de la naturaleza de la fuente y del tipo de información recabada. Estos datos pueden consultarse en publicaciones precedentes de los autores.

3 Cinco mujeres y cinco hombres cuya identidad se preserva mediante el uso de nombres de deidades griegas como pseudónimo. Tres de ellos con estudios primarios, cinco con educación secundaria y dos con estudios universitarios. Todos ellos en desempleo durante más de un año. 
Triple Triangulación Compleja

\section{Referencias}

ALCOFORADO, Luís. Estrategias, Retos y Recursos para los Orientadores en el Escenario de la Educación y Formación a lo Largo de la Vida. In: FIGUERA, Pilar (Org.). Orientación Profesional y Transiciones en el Mundo Global. Barcelona: Laertes, 2013. P. 21-50.

ÁLVAREZ-GÁLVEZ, Javier; SUÁREZ-VERGNE, Álvaro. Estudio de la Asociación Entre la Discriminación Laboral, la Salud y el Uso de Servicios Sanitarios en Andalucía: una aplicación de propensity score matching con muestras pequeñas. EMPIRIA. Revista de Metodología de Ciencias Sociales, n. 48, p. 147-166, 2020. DOI: <https://doi.org/10.5944/empiria.48.2020.28074>.

AMBER, Diana; DOMINGO, Jesús. La presencia y representación del desempleo de los mayores de 45 años en la prensa española. Observatorio (OBS*) Journal, Lisboa, v. 9, n. 4, p. 085-091, 2015. DOI: <http://dx.doi.org/10.15847/obsOBS942015906>.

AMBER, Diana; DOMINGO, Jesús. Los lunes al sol. Retrato social de historias de vida silenciadas. Cultura, lenguaje y representación, Castellón de la Plana, v. XVI, p. 21-36, 2016. DOI: <http://dx.doi.org/10.6035/clr.2016.16.2>.

AMBER, Diana; DOMINGO, Jesús. Desempleo en mayores de 45 años. ¿ Un bucle sin salida? Revista Brasileira de Orientação Profissional, São Paulo, v. 18, n. 2, p. 195-207, 2017a. DOI: <http://dx.doi.org/10.26707/1984-7270/2017v18n2p195>.

AMBER, Diana; DOMINGO, Jesús. What Statistics Hide? Secondary Analysis of Unemployment in Spain. International Journal of Euro-Mediterranean Studies, Slovenia, v. 10, n. 2, p. 29-47, 2017b.

AÑEZ, Carmen. Flexibilidad Laboral iFin del Trabajo Permanente? Telos: Revista de Estudios Interdisciplinarios en Ciencias Sociales, v. 18, n. 2, p. 250-265, 2016.

ARESE, César. El 'Edadismo' Laboral y Previsional. Revista Derecho de las Minorías, Córdoba, v. 3, p. 138-163, 2020. DOI: <http://dx.doi.org/10.22529/ rdm.2020(3)05>.

ARRÁIZ-PÉREZ, Ana; SABIRÓN-SIERRA, Fernando; SUÁREZ-ORTEGA, Magdalena. Personas Emprendedoras: vidas ejemplares y claves educativas para la reorientación de la carrera. Qualitative Research in Education, v. 9, n. 2, p. 217247, 2020. DOI: <http://dx.doi.org/10.17583/qre.2020.5395>.

BAUMAN, Zygmunt; LEONCINI, Thomas. Generación Líquida: transformaciones en la era 3.0. Barcelona: Paidós, 2018.

BECK, Ulrich. Varieties of Second Modernity and the Cosmopolitan Vision. Theory, Culture \& Society, n. 11, p. 257-270, 2016. DOI: <https://doi. org/10.1177/0263276416671585>.

BECK, Ulrich; Beck-Gernscheim, Elisabeth. La Individualización: el individualismo institucionalizado y sus consecuencias sociales y políticas. Barcelona: Paidós, 2003.

BERNAD, Joan; MARTÍNEZ, Ignacio; MOLPECERES, Mariángeles. Trabajo, Precariedad y Ciudadanía. Las Políticas Sociolaborales en el Contexto del Desmantelamiento de la Sociedad Salarial. In: CÓRDOBA, Ana; MARTíNEZ, Ignacio (Org.). Trabajo, Empleabilidad y Vulnerabilidad Social: condicionantes y potencialidades de la integración a través de las empresas de inserción social. Valencia: Universitat de Valencia, 2011. P. 23-60.

BOLÍVAR, Antonio; DOMINGO, Jesús. La Investigación (Auto)Biográfica y Narrativa en Educación. Barcelona: Octaedro, 2019. 
CABALLOL, José M. ¿Inempleables o Improductivos? Madrid: Asociación RAIS, 2002.

CALVO, Francisco Javier. Los Mayores en el Nuevo Derecho del Empleo. In: RODRÍGUEZ-PIÑERO, Miguel; CASTELLANO, Emilia (Org.). Transiciones Laborales y Reformas del Mercado de Trabajo: su influencia sobre el empleo de jóvenes y mayores. Albacete: Editorial Bomarzo, 2014. P. 333-423.

CHEUNG, Chau-Kiu.; KAM, Ping; NGAN, Raymond. Age Discrimination in the Labour Market From the Perspectives of Employers and Older Workers. International Social Work, Australia, v. 54, n. 1, p. 118-136, 2011. DOI: <https://doi. org/10.1177/0020872810372368>.

CLIMENT-RODRÍGUEZ, José Antonio; NAVARRO-ABAL, Yolanda. Nuevos Retos en Orientación Laboral: de itinerarios personales de inserción a la construcción de marcas profesionales. Revista Española de Orientación y Psicopedagogía, v. 27, n. 2, p. 126-133, 2016.

CLIMENT-RODRÍGUEZ, José Antonio; NAVARRO-ABAL, Yolanda; LÓPEZ-LÓPEZ, María José; GÓMEZ-SALGADO, Juan; APARICIO-GARCÍA, Marta Evelia. Grieving for Job Loss and Its Relation to the Employability of Older Jobseekers. Frontiers in Psychology, v. 10, p. 1-12, 2019. DOI: <https://doi.org/10.3389/fpsyg.2019.00366>.

CRESWELL, John. Educational Research: planning, conducting, and evaluating quantitative and qualitative research. Upper Saddle River: Pearson Education, 2005.

DENZIN, Norman Kent; LINCOLN, Yvonna Sessions. The Sage Handbook of Qualitative Research. Thousand Oaks: Sage, 2011.

DUBAR, Claude. La Crisis de las Identidades. La interpretación de una mutación. Barcelona: Ediciones Bellaterra, 2002.

ERIKSON, Erik Homburger. El Ciclo Vital Completado. Edición revisada y ampliada. Buenos Aires: Paidós, 2000.

ESPAÑA. Real Decreto-Ley 3/2011, de 18 de febrero, de medidas urgentes para la mejora de la empleabilidad y la reforma de las políticas activas de empleo. Boletín Oficial del Estado, Madrid, n. 43, Sec. I, p. 19240-19260, 19 feb. 2011.

ESPAÑA. Real Decreto 694/2017, de 3 de julio, por el que se desarrolla la Ley 30/2015, de 9 de septiembre, por la que se regula el Sistema de Formación Profesional para el Empleo en el ámbito laboral. Boletín Oficial del Estado, Madrid, n. 159, Sec. I, p. 56864-56899, 5 jun. 2017FELBER, Christian. La Economía del Bien Común. Barcelona: Deusto, 2012.

FLICK, Uwe. Introducción a la Investigación Cualitativa. Madrid: Ediciones Morata, 2007.

FLICK, Uwe. Designing Qualitative Research. Thousand Oaks: Sage, 2018a.

FLICK, Uwe. Doing Triangulation and Mixed Methods. Thousand Oaks: Sage, 2018b.

GARCÍA-CALAVIA, Miguel Ángel. The COVID-19 Shows Problems of the Social Reality of Work. Revista Española de Sociologia, v. 29, n. 3, p. 727-736, 2020. DOI: $<$ https://doi.org/10.22325/fes/res.2020.46>.

GUNDERSON, Morley. Age Discrimination and Employment in Canada. Contemporary Economic Policy, United Kingdom, v. 21, n. 3, p. 318-329, 2003.

HEATH, Linda. Triangulation: Methodology. In: SMELSER, Neil; BALTES, Paul (Org.). International Encyclopedia of the Social and Behavioral Sciences. Amsterdan: Elsevier Science, 2001. P. 15901-15906.

Educação \& Realidade, Porto Alegre, v. 46, n. 1, e106754, 2021. 
Triple Triangulación Compleja

HOHMANN, Reinhard. Lebenslänglich Lernen: satiren aus der weiterbildung. Münster: ATE Edition, 2004.

INSTITUTO NACIONAL DE ESTADÍSTICA. Resultados Nacionales. Parados. Madrid: INE BASE, 2020. Disponible en: <http://www.ine.es/dynt3/inebase/es/ index.htm? padre=982\&capsel $=986>$ Acceso en: 10 ago 2020 .

IZQUIERDO, Tomás; LÓPEZ-MARTÍNEZ, Olivia. El Rol de las Actitudes en la Inserción Laboral de los Desempleados Mayores de 45 Años. Universitas Psychologica, Bogotá, v. 12, n. 3, p. 911-922, 2013. DOI: <http://dx.doi.org/10.11144/ Javeriana.UPSY12-3.rail>.

JIMÉNEZ, Jorge. El Efecto Mateo: un concepto psicológico. Papeles del Psicólogo, Madrid, v. 30, p. 145-154, 2009.

LASSUS, Lora; LOPEZ, Steven; ROSCIGNO, Vincent. Aging Workers and the Experience of Job Loss. Research in Social Stratification and Mobility, United Kingdom, n. 41, p. 81-91, 2015. DOI: <http://dx.doi.org/10.1016/j.rssm.2015.01.001>.

MACHADO, Antonio. Campos de Castilla. Salamanca: Ediciones Anaya, 1965.

MARTÍNEZ, Laureano; BOGINO, Victoria. La Instrumentación en España de las Políticas Europeas de Emprendimiento ¿creación de empleo o profundización de la crisis del empleo asalariado? Revista Eletrônica de Ciência Política, Paraná, v. 6, n. 1, p. 187-208, 2015. DOI: <http://dx.doi.org/10.5380/recp.v6i1.37493>. MORIN, Edgar. Introducción al Pensamiento Complejo. Barcelona: Gedisa, 2008.

OLESYA, Savelyeva. Labor Ageism as a Form of Social Behavior and its Consequence. In: VIKTOROVNA, Posnova M. (Org.). Modern Socioeconomic Processes: experience of theoretical and empirical analysis. Petrozavodsk: International Center for Scientific Partnership 'New Science', 2020. P. 113-136

POCHMANN, Marcio. Trabalho e formação. Educação \& Realidade, Porto Alegre, v. 37, n. 2, p. 491-508. DOI: <https://doi.org/10.1590/S2175-62362012000200009>. RODRÍGUEZ-RODRÍGUEZ, Ignacio. La `nave espacial Tierra’ de Kenneth Boulding. Revista de Economía Crítica, Madrid, n. 14, p. 320-326, 2012.

RUESGA, Santos; DA SILVA, Julimar; MONSUETO, Sandro. Estudiantes Universitarios, Experiencia Laboral y Desempeño. Revista de Educación, Madrid, n. 365, p. 67-95, 2014. DOI: <http://dx.doi.org/10.4438/1988-592X-RE-2014-365-265>.

SALVADOR, Vicent; SAMPIETRO, Agnese. Understanding the Discourse of Aging: a multifaceted perspective. Newcastle upon Tyne: Cambridge Scholars Publishing, 2020.

SÁNCHEZ, María. La Orientación Laboral en Contextos de Diversidad Personal, Social y Cultural. In: SOBRADO, Luis; CORTÉS, Alejandra (Org.). Orientación Profesional. Nuevos escenarios y perspectivas. Madrid: Biblioteca Nueva, 2009. P. 161-183.

SANTANDER, Pedro. Por Qué y Cómo Hacer Análisis de Discurso. Cinta de Moebio, Santiago, n. 41, p. 207-224, 2011. DOI: <http://dx.doi.org/10.4067/S0717554X2011000200006>.

SCHÖMANN, Klaus. Ungenutzte Potenziale bei Übergängen zwischen Bildung und Arbeit: ein internationaler vergleich. Wirtschaftsdienst, Berlin, n. 91, p. 10 14, 2011. DOI: <http://dx.doi.org/10.1007/s10273-011-1177-y>.

SELL, Lucía; MARTÍNEZ-PECINO, Roberto; LOSCERTALES, Felicidad. El Cine como Herramienta Educativa para Abordar la Violencia en las Aulas. Píxel-Bit. Revista de Medios y Educación, Sevilla, n. 45, p. 111-124, 2014. DOI: <http:// dx.doi.org/10.12795/pixelbit.2014.i45.08>. 
SENNETT, Richard. La Corrosión del Carácter. Las consecuencias personales del trabajo en el nuevo capitalismo. Barcelona: Anagrama, 2006.

SERRAT, Rodrigo; VILLAR, Feliciano; GIULIANI, María-Florencia; ZACARÉS, Juan-José. Older People's Participation in Political Organizations: the role of generativity and its impact on well-being. Educational Gerontology, v. 43, n. 3, p. 128-138, 2017. DOI: <https://doi.org/10.1080/03601277.2016.1269541>.

STAKE, Robert. Evaluación Comprensiva y Evaluación Basada en Estándares. Barcelona: Graó, 2006.

STAKE, Robert. Multiple Case Study Analysis. New York: Guilford Press, 2013. SUBIRATS, Joan. Pobreza y Exclusión Social. Un análisis de la realidad española y europea. Barcelona: Fundación La Caixa, 2004.

TEJADA, Jose; GIMÉNEZ, Vicente. Formación de Formadores. Escenario institucional. Madrid: Thomson-Paraninfo, 2007.

THIEME, Paula; BRUSCH, Michael; BÜSCH, Victoria; STAMOV, Christian. Work Context Influences on Older Workers' Motivation for Continuing Education. Zeitschrift für Erziehungswissenschaft, Berlin, v. 18, n. 1, p. 71-87, 2015. DOI: <http://dx.doi.org/10.1007/s11618-014-0600-8>.

VALDEZ-MONTESDEOCA, Frank; TAPIA-ESPINOZA, Nancy. La Edad como Factor Limitante en la Inserción Laboral desde la Perspectiva Empresarial. Revista 593 Digital Publisher CEIT, v. 5, n. 2, p. 164-179, 2020. DOI: <https://doi.or g/10.33386/593dp.2020.2.215>.

VAN DIJK, Teun Adrianus. Discurso y Poder. Barcelona: Gedisa, 2009.

WELLER, Sally. Discrimination, Labour Markets and the Labour Market Prospects of Older Workers: what can a legal case teach us? Work, Employment and Society, Londres, v. 21, n. 3, p. 417-437, 2007. DOI: <http://dx.doi. org/10.1177/0950017007080006>.

ZACARÉS, Juan José; SERRA, Emilia. Explorando el Territorio del Desarrollo Adulto: la clave de la generatividad. Cultura y Educación, Madrid, v. 23, n. 1, p. 75-88, 2011. DOI: <http://dx.doi.org/10.1174/113564011794728533>.

Diana Amber es Profesora del Departamento de Pedagogía de la Universidad de Jaén. Doctora con mención internacional por la Universidad de Granada. Su principal línea de investigación se centra en la formación para el empleo de colectivos vulnerables. Miembro del grupo de investigación FORCE y de la Red RIDIPD.

ORCID: http://orcid.org/0000-0002-9765-3547

E-mail: damber@ujaen.es

Jesús Domingo es Catedrático de la Universidad de Granada. Doctor en Ciencias de la Educación, especialista en formación, asesoría y liderazgo para la inclusión de colectivos vulnerables, la mejora de la educación pública y la justicia social. Miembro del grupo de investigación FORCE, la Red de Investigación RILME y el Proyecto Atlántida.

ORCID: http://orcid.org/0000-0002-8319-5127

E-mail: jdomingo@ugr.es

Editor a cargo: Beatriz Vargas Dorneles 
Este é um artigo de acesso aberto distribuído sob os termos de uma Licença Creative Commons Atribuição 4.0 Internacional. Disponível em: <http:// creativecommons.org/licenses/by/4.0>. 